\title{
ПОЗВОНОЧНО-СПИННОМОЗГОВАЯ ТРАВМА У ДЕТЕЙ, ПОСТРАДАВШИХ ВНУТРИ АВТОМОБИЛЯ ПРИ ДОРОЖНО-ТРАНСПОРТНЫХ ПРОИСШЕСТВИЯХ
}

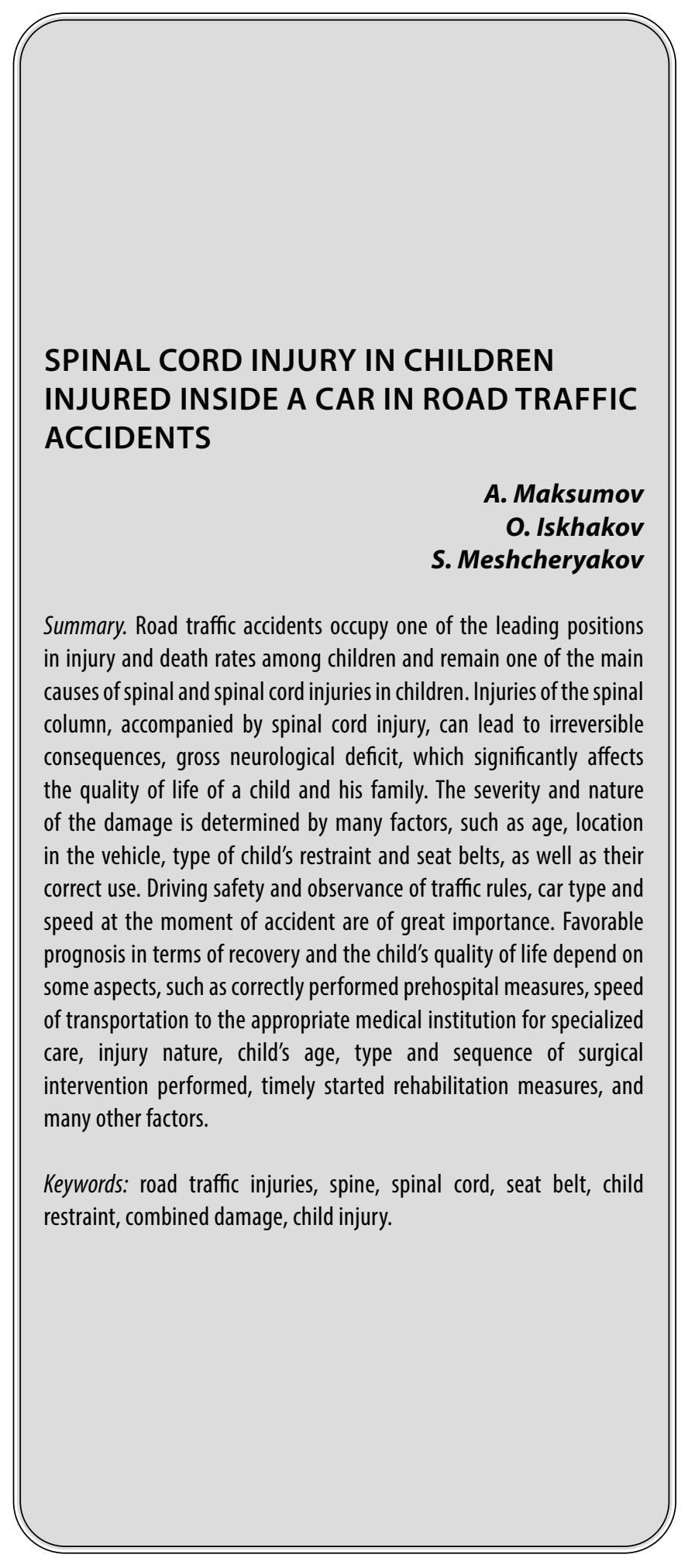

Максумов Александр Алишерович

Н.с., нейрохирург, ГБУз НИИ «Неотложной детской хирургии и травматологии» ДЗМ dr.maksumov@gmail.com

Исхаков Олимджан Садыкович

Д.м.н., нейрохирург, ГБУз НИИ «Неотложной детской хирургии и травматологии» ДЗМ

Мещеряков Семен Владимирович

Н.с., нейрохирург, ГБУз НИИ «Неотложной детской хирургии и травматологии» ДЗМ

Аннотация. Дорожно-транспортные происшествия занимают одну из лидирующих позиций по травматизму и смертности среди детей, а также остаются одной из основных причин травм позвоночника и спинного м0зга у детей.

Повреждения позвоночного столба, сопровождаемые травмой спинного мозга, могут приводить к необратимым последствиям, грубому неврологическому дефициту, что значительно влияет на качество жизни ребенка и его семьи. Тяжесть и характер повреждения определяется многими факторами, такими как возраст, местоположение в автомобиле, тип детского удерживающего устройства и ремней безопасности, правильностью их использования. Большое значение имеют безопасность вождения и соблюдение правил дорожного движения, тип автомобиля, скорость, на которой произошла авария.

Для благоприятного прогноза в плане выздоровления и жизни ребенка в целом имеют такие аспекты, как правильно выполненные догоспитальные мероприятия, скорость доставки в соответствующее лечебное учреждение для оказания специализированной помощи, характер травмы, возраст ребенка, вид и последовательность выполняемого оперативного вмешательства, своевременность проведения реабилитационных мероприятий и многие другие показатели.

Ключевые слова: дорожно-транспортный травматизм, позвоночник, спинной мозг, ремни безопасности, детские удерживающие устройства, сочетанные повреждения, детская травма. 


\section{Ввемение}

$\mathbf{0}$ дной из глобальных мировых проблем является дорожный травматизм [1,2]. Рост автотранспорта и одновременное увеличение количества личных автомобилей приводит к сохранению высоких показателей травматизма при дорожно-транспортных происшествиях (ДТП), в том числе среди детей $[2,3]$.

ДТП является одной из основных повреждения позвоночника и спинного мозга в детском возрасте [3,4]. Большое число повреждений с вовлечением позвоночника и невральных структур у детей при нахождении внутри автомобиля носят характер сочетанной травмы. Существенное влияние на характер повреждения спинного мозга, клинические проявления, выбор тактики ведения пациентов оказывают возрастные анатомо-физиологические особенности позвоночника и спинного мозга у детей, а также структура и тяжесть сочетанных повреждений.

Актуальность изучения позвоночно-спинномозговой травмы (ПСМТ) в структуре сочетанных повреждений у детей, находившихся в салоне автомобиля обусловлена прежде всего тем, что тяжелые повреждения внутренних органов, деформация позвоночника, тяжелые неврологические нарушения приводят к инвалидизации в детском возрасте.

Цель работы. Анализ литературных данных, посвященный вопросам позвоночно-спинномозговой травмы у детей, находившихся внутри автомобиля при ДТП и использовавших ремни безопасности (РБ) и детские удерживающие устройства (ДУУ), который может помочь в построение правильного лечебно-диагностического алгоритма.

\section{ЭпиАемиология травмы} позвоночника у Аетей

Согласно исследованиям, проведенным в Австралии, Объединенных Арабских Эмиратах и Ирландии, ДТП были основной причиной ПСМТ у взрослого населения и составили $45 \%$, 48\% и $34-42 \%$, соответственно $[27 ; 28 ; 29 ; 30]$. Анализ аварийности в Российской Федерации показывает снижение числа аварий с 173700 до 133203 в период от 2016 по 2018 годы. Но при анализе показателей смертности за 2018 г. отмечается рост летальных исходов на 16000 в сравнении с показателями за 2015-2016 гг. Самой частой причиной ДТП остается несоблюдение скоростных режимов $(31,4 \%)$ [31]. Детский травматизм, обусловленный ДтП, относится к достаточно актуальной проблеме в РФ, причем самый частый вид травмы, который сопровождается высокими показателями смертности - это травма, получен- ная внутри автомобиля. [8]. По данным ГИБДД только за первый месяц 2018 г. произошло 1196 ДТП с участием детей, 57,5\% из которых получили травму в салоне автомобиля [32]. Согласно исследованию в США проведённомy R. Zonfrillo, M. Locey и соавторов, в исследование которых вошли 59 детей пострадавших в ДТП и имевших в структуре травмы спинальные повреждения. Пострадавшие использовали различные средства фиксации: обычный трехточечный ремень $88 \%$, кресло с пятиточечным ремнем, установленным по ходу движения $12 \%$ [10].

\section{Эпихемиология травмы позвоночника у метей}

Согласно ВО3, выделяют следующие возрастные групп детей: I группа от 0 до 3 лет, II группа от 3 до 6 лет, III группа от 6 до 10 лет, IV группа от 10 до 15 лет и V группа от 15 до 18 лет. В каждой из возрастных групп используются различные ДУУ, которые представлены в таблице 1 , рекомендованные для перевозки детей. Лица старше 12 лет используют стандартные трехточечные ремни безопасности.

Большое значение в характере повреждений позвоночника и спинного мозга у детей отводится факторам, обусловленным возрастными анатомо-морфологическими особенностями организма ребенка, специальными дуУ с различными вариантами фиксации ребенка, месту и положению ребенка в салоне $[5,6]$ Автотранспортной экспертизой США установлен тип ДТП, при котором возможно повреждение ремнем безопасности - лобовое столкновение, боковой удар и опрокидывание. Такие повреждении возможны уже при сред-

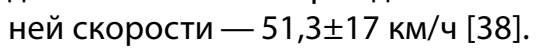

В структуре повреждений у детей можно выделить черепно-мозговую травму, показатели которой варьируются в зависимости от возраста детей и составляют от 54\% у детей в возрасте $10-15$ лет, до $88 \%$ у детей до 3-х лет. Особенности повреждений туловища определяются возрастными показателями и типами используемой фиксации внутри автомобиля. Для детей 3-15 лет характерны переломы грудной клетки, травмы легких различной степени тяжести, переломы позвоночника в грудном отделе, которые редко возникают в возрасте до 3-х лет. У детей в возрастной группе 10-15 лет наиболее часто возникают переломы костей таза, тяжелые повреждения органов брюшной полости в сочетании с переломами позвоночника на поясничном уровне. Скелетной травме верхних конечностей чаще подвергаются дети 3-6 лет. Травма нижних конечностей чаще встречаются у детей в I и II возрастных группах, однако повреждения костей голени чаще встречается у детей в старшем возрасте. Подобное 
Таблица 1. Детские удерживающие устройства.

\begin{tabular}{|c|c|c|c|}
\hline Группа & Особенности фиксации & Возраст & Вес и рост \\
\hline $0+$ & Автолюлька & 0-6 мес. & до 13 кг; 40-85 см \\
\hline 1 & Пятиточечный ремень, спинка, боковая защита & 9мес - 4 года & $9-18$ кг; 80-105 см \\
\hline 2 & Крепление штатными трёхточечными ремнями & 3,5-7 лет & $15-26$ кг; $100-145 \mathrm{~cm}$ \\
\hline 3 & Бустер & 6-12 лет & до 36 кг; до 154 см \\
\hline
\end{tabular}

\section{B2. Posterior tension band disruption}

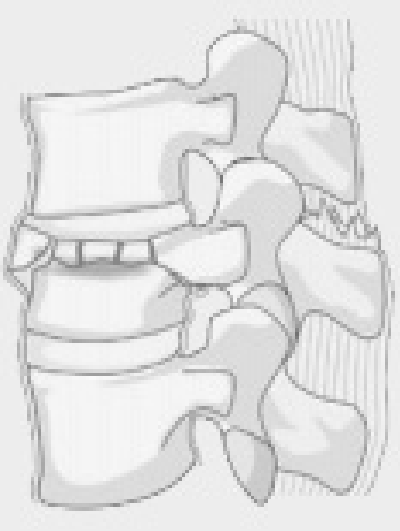

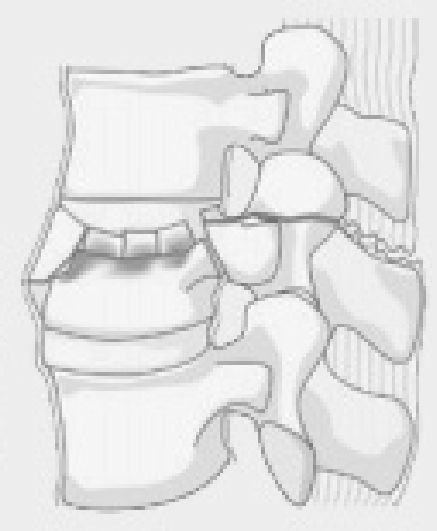

Рис. 1. AO Spine: тип B

\section{B1. Transosseous tension band disruption / Chance fracture}

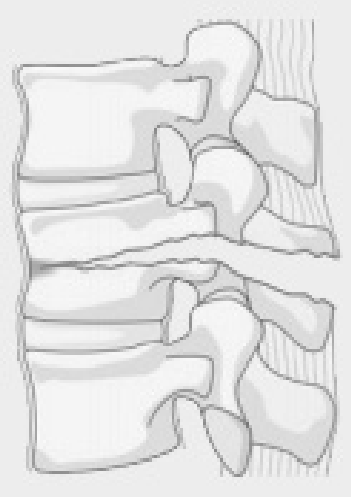

распределение травм нижних конечностей связано с особенностями роста и положения ног в автомобиле в совокупности с ДУУ и РБ. R. Zonfrillo отмечает, что позвоночный столб - самая уязвимая область, на 42 ребенка - 94 повреждения, следовательно у большинства детей были многоуровневые и множественные повреждения позвоночного столба. В структуре травмы также встречались повреждения нижней конечности или таза - 56 повреждений, органов брюшной полости - 39, органов грудной клетки - 36 [10]. Поскольку соотношение головы и туловища у детей больше, по сравнению со взрослыми, то точка приложения травмы смещается к голове и, следовательно, увеличивается риск затылочно-атлантоаксиального повреждения. Отсутствие полной оссификации и слабых связок увеличивает риск повреждения связок [7,8,9,10].

Основные механизмы, принимаюшие участие в повреж ении позвонков и спинного мозга у Аетей при $\triangle T П$ с использованием среАств фиксашии

Впервые, Tourin B. и Garrett J. описывают и вводят понятие «Seat-belt» травмы (травма от ремня безо- 


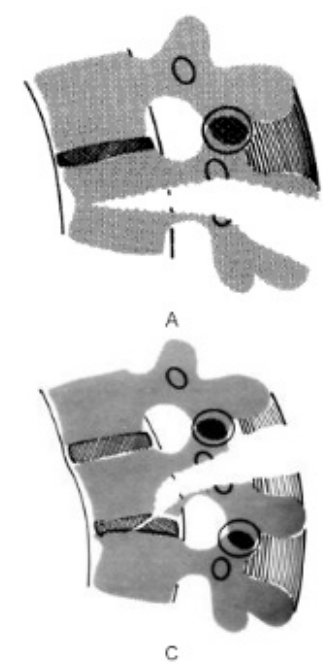

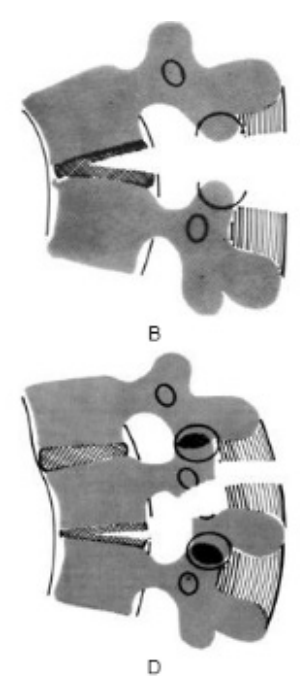

Pис. 2. Dennis

пасности) в 1960. Обратившие внимание на специфические кожные метки - широкополосные ссадины и кровоподтеки на туловище, соответствующие лямкам ремня [25]. Такие повреждения получили название «Seat-belt». Это повреждения от ремня безопасности, возникающее в момент столкновения автомобиля, и характеризующееся повреждением мягких тканей и внутренних органов в результате сжатия их петлей ремня и повреждением позвоночного столба в результате флексии и деструкции относительно точки фиксации. Смогласно классификации AO Spine это повреждения тип В1 и В2 (Рис. 1) [36]. Согласно классификации Dennis - флексионно-дистракционное повреждение (Рис. 2) [37].

Как правило, механизмы, приводящие к такого рода травмам, являются комбинированными. Наиболее частыми являются: компрессионная флексия; дистракционная флексия (комбинация сгибания и растяжения), торсионная (вращающая, ротационная) флексии, дистракционная экстензия (комбинация разгибания и растяжения) $[13,15]$.

К основному патофизиологическому механизму можно отнести две фазы: первая - повреждений спинного мозга относят немедленное механическое повреждение, которое способствует развитию ушибов в области спинного мозга вследствие постоянного или временного сдавливания. Развитие второй фазы обусловлено появлением дисфункциональных расстройств и гибелью клеток нервной ткани через несколько часов или недель после первичного повреждения в результате деструктивного и биохимического воздействия. В развитии нарушений проводимости со стороны спинного мозга важная роль отводят процессам, формирующимся в остром периоде травмы спинного мозга. Такие повреждения приводя к необратимым изменениям в тканях и к появлению грубого неврологического дефицита $[11,12,13]$. Существует большое количество работ, посвящённых синдрому ремня безопасности (seat-belt syndrome (SBS)), но большинство из них не затрагивает проблему травм позвоночника и сфокусированы в основном на повреждениях грудной клетки, органов брюшной полости, скелетной травме $[39,40]$.

\section{А^горитм АИагностИки ПСМТ}

В клинической практике у детей наиболее часто используется следующая последовательность диагностических мероприятий [16]: 1) Rg-графия позвоночного столба. 2) Спондилография. 3) КТ позвоночного столба на уровне повреждения. 4) Люмбальная пункция с ликвородинамическими пробами. 5) КТ-миелография. 6) МРТ. 7) Выполнение вертебральной ангиографии.

Результаты проведенного МРТ исследования позволяют оценить повреждения связочного, мышечного и дискового аппарата позвоночника, выявить наличие стеноза позвоночного канала, сдавление дурального мешка, гемодинамические нарушения, повреждения спинного мозга и нервных корешков [41].

Многообразие сочетанных повреждений и их отсроченные клинические проявления, неполная инструментальная диагностика приводят к тому, что от $25 \%$ до 37\% повреждений пропускается. Акцент делается на ведущем повреждении, тогда как другие сочетанные повреждения, на первый взгляд малозначимые, игно- 
Таблица 2. Сравнение MIS и отрытой ТПФ.

\begin{tabular}{|c|c|c|c|}
\hline & MIS & Открыто & $p$ \\
\hline \multicolumn{4}{|l|}{ Пол } \\
\hline M & 11 & 18 & \\
\hline ж & 0 & 9 & \\
\hline Возраст & $40,1+/-20.3$ & $24,7+/-15.3$ & 0,4 \\
\hline \multicolumn{4}{|l|}{ ASIA } \\
\hline до операции & 5 & $4,41+/-1.28$ & 0,31 \\
\hline в раннем периоде & 5 & $4,56+/-1.19$ & 0,42 \\
\hline Койко-дни & $7,6+/-3.8$ & $11,2+/-7$ & 0,12 \\
\hline
\end{tabular}

рируются [10]. Согласно исследованию Lindsay M и соавторов из анализа лечения 26 детей, с нестабильными повреждениями торако-люмбального отдела, где количество детей с переломами типа Chance (seat-belt) было 26\%. В 8\% эти повреждения были выявлены лишь спустя два месяца, когда имело место нарастание деформации в сегменте повреждения [33]. Например, в случае повреждения органов брюшной полости с внутрибрюшным кровотечением и необходимости срочного хирургического вмешательства нет возможности выполнить в полном объеме диагностический этап, тяжесть состояния или медикаментозная седация не позволяют оценить неврологический статус и выполнить томографическое исследование. По данным зарубежных авторов, в $29 \%$ случаев во время диагностики были пропущены переломы поясничного отдела позвоночника, так как общепринятая рутинная рентгенография, в отношении детей часто малоинформативна. С другой стороны, сила, с которой ремень безопасности прижимает полый орган (кишечник), практически всегда приводит к травматизации и возможной ишемии стенки кишки, что может привести к отсроченной перфорации кишки и перитониту [13]. В некоторых описанных случаях, явления некроза стенки кишки и перфорации полого органа возникали через 12-24 часа после травмы, что усугубляет состояние пациента. Совокупность данных факторов приводит к поздней диагностике ПСМТ у детей [12].

\section{Определение тактики консервативного и хирургического

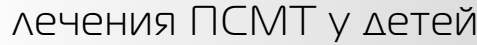

Большинство повреждений у детей полученных в ДТП с использованием средств фиксации сопровождается обильной кровопотерей и шоком, что обусловлено тяжестью полученной сочетанной травмы. Особенно это характерно при тяжелой ЧМТ и повреждением паренхиматозных органов в сочетании с ПСМТ [29].
Целью консервативного лечения позвоночно-спиномозговой травмы переломов является ранняя и полная разгрузка поврежденного отдела позвоночника. Это выполняется при неосложнённой травме и зависит от уровня повреждения. Для шейного или верхнегрудного отдела это может быть вытяжение петлей Глиссона [17]. Консервативное лечение шейного отдела позвоночника может включать внешнюю стабилизацию с помощью мягкого шейного воротника, полужесткого воротника или фиксирующих устройств. Для грудного и поясничного отдела могут быть использованы различные варианты корсетов. Так же широко используются лечебная физкультура, массаж и физиотерапия. [26].

Хирургическое лечение в основном показано при нестабильных повреждениях, невправимых переломах или вывихах, прогрессирующем неврологическом дефиците в результате компрессии и прогрессирующей деформации. В настоящее время в практике хирургического лечения детей с ПСМТ применяют хирургические методы лечения такие же как у взрослых [18].

Основное количество публикаций посвящены оперативному лечению нестабильных и осложненных переломов позвоночника у детей. В их число водят операции с комбинированными доступами. [18,19]. При выраженных деформациях позвоночного столба необходимо решить все ортопедические задачи в остром периоде. [19]. Как сообщают A. Alexandre и соавторы в анализе оперативного лечения 35 детей, с переломами типа В1 и В2 в результате ДТП с использованием фиксации у детей, в динамике имеет место нарастание кифотической деформации в 25\%, что потребовало задней фиксации в отсроченном периоде [37]. У детей пострадавших при использовании РБ и дУУ в тяжелом состоянии, с сочетанными повреждениями органов брюшной полости и других систем невозможно выполнить хирургическое пособие в необходимом объёме и в рекомендуемы сроки $[19,20]$. Большое внимание ис- 
следователей уделяется срокам, прошедшим с момента получения травмы. Если от момента травматического воздействие прошло до 10 дней, то пациентам, имеющим нестабильные переломы позвоночник, чаще производят выполнение репозиционно-стабилизирующей операции из заднего доступа. В случае, если от момента травмы прошел больший срок, необходимо выполнение реконструкции переднего и среднего опорного комплекса при помощи корпородеза, а затем выполнение стабилизации металлоконструкцией [20].

В случае травматизации в грудном отделе позвоночника у детей используют стабилизацию и редукцию при помощи крючковой системой. При переломе тела одного позвонка в поясничном отделе проводят бисегментарную транспедикулярную репозицию и фиксацию. Одним из динамически развивающихся направлений спинальной хирургии является лечение переломов краниовертебрального уровня [20,21,22]. В том случае, если у пациента диагностировано наличие ПСМТ, сопровождаемого грубыми неврологическими расстройствами, необходимо проведение декомпрессивно-стабилизирующей операции в первые 6-9 часов от момента травмы, что остается проблематично у пациентов с тяжелой сочетанной травмой [22]. В исследовании Mariam S. и соавторов показано что при анализе лечения и отдаленных результатов 8 детей пострадавших в ДТП и использованием ДУУ и РБ, и имевшим тяжелые сочетанных повреждения включая ПСМТ, в 50\% случаев дети имели грубые неврологические нарушения, которые не регрессировали, что привело к инвалидизации пациентов в детском возрасте [34].

Таким образом к основным этапам оперативного лечения ПСМТ у детей можно отнести следующие:

- Выполнение редукционно-стабилизующей операции из заднего доступ при помощи ламинарных и педикулярных крючков, транспедикулярных винтов.

- Задний локальный спондилёз.

- В случае смещения отломков тел позвонков в позвоночный канал необходимо их удаление из переднебокового доступа;

Реконструкция передних отделов из переднебокового доступа с использование сетчатых имплантов, алокости или аутокости. [22,23].

Согласно данным литературы, можно выделить три наиболее перспективных направления развития спинальной хирургии в лечении травматических переломов нижне-шейного, грудного и поясничного отделов позвоночника:

1. чрезкожные методики фиксации и\или стабилизации сломанных позвонков;
2. минимально инвазивные доступы;

3. эндоскопические технологии.

A. Grossbach и соавторы провели исследование результатов лечения 38 пациентов детского возраста с повреждением Chance. В 27 случаях выполнялась открытое оперативное вмешательство с задней фиксацией. В 11 случаях выполнена малоинвазивная, перкутанная фиксация. Проведен сравнительный анализ ближайших результатов (таб. 2). По результатам исследования количество проведенных койко-дней при использовании малоинвазивной транспедикулярной фиксации сокращается практически в два раза в сравнении с открытой операцией [35].

Несмотря на изучение различных аспектов проблемы ПСМТ и других тяжелы повреждений у детей связанных с РБ и ДУУ и небольшое количество работ в данной области, единых подходов к хирургическому лечению травмы позвоночника у детей в настоящее время не разработано и подходы к лечению определяются индивидуально с учетом клинической картины, тяжести травмы, степени неврологических нарушений, техническими возможностями лечебного учреждения [25]. Кроме того, важное значение для благоприятного прогноза в плане выздоровления и жизни в целом имеют такие аспекты, как правильно выполненные догоспитальные мероприятия, скорость доставки в соответствующее лечебное учреждение для оказания специализированной помощи, характер травмы, возраст ребенка, вид выполняемого оперативного вмешательства, наличие или отсутствие послеоперационных осложнений, своевременность проведения реабилитационных мероприятий и многие другие показатели. [20,21,22,23]

\section{$\mathrm{BbBO \triangle}$}

Актуальность проблемы ПСМТ у детей пострадавших внутри автомобиля на сегодняшний день не вызывает сомнений. Поражение спинного мозга и его элементов вследствие травматического сдавления отломками тела позвонка приводит к утяжелению клинической ситуации. Многообразие сочетанных повреждений других органов и систем. Приводит к поздней диагностике и невозможности выполнения оперативного лечения ПСМТ в раннем периоде.

У детей с осложненной и нестабильной ПСМТ может развиваться стойкий, необратимый неврологический дефицит, который сохраняется на протяжении всей жизни и приводят к стойкой инвалидизации пациента. Это в свою очередь, оказывает непосредственное влияние на жизнь и развитие ребенка и качество жизни его семьи. Разработка последовательности лечебно-диагностического алгоритма улучшит результаты лечения детей с подобными повреждениями. 


\section{ЛИТЕРАТУРА}

1. Goniewicz K, Goniewicz M, Pawłowski W, Fiedor P. Children Road traffic injuries in Poland. Polish Journal of Public Health. 2017;127: 44-48. doi:10.1515/ pjph-2017-0010.

2. Yin Z, Wu J, Luo J. Burden and trend analysis of injury mortality in China among children aged 0-14 years from 2004 to 2011. BMJ 0pen. 2015;5: e007307.

3. Development and validation of a self-administered questionnaire to estimate the distance and mode of children's travel to school in urban India. BMC Med Res Methodol. 2015; 15:92. doi:10.1186/s12874-015-0086-y

4. Икоева Г.А., Виссарионов С.В., Баиндурашвили А.Г., Кокушин Д.Н., Овечкина А.В., Солохина И.Ю., и др. Сравнительный анализ динамики неврологических нарушений у детей с позвоночно-спинномозговой травмой поясничного отдела в зависимости от сроков хирургического вмешательства. Международный журнал прикладных и фундаментальных исследований. 2018;5-1:97-103;

5. Савенкова Е.Н., Ефимов А.А., Семижонова В.Н., Купрюшин А.С. К вопросу о возрастных особенностях детского автомобильного травматизма. Известия высших учебных заведений. Поволжский регион. Медицинские науки. 2014; 3:127-134.

6. Савенкова Е.Н., Ефимов А.А., Алексеев Ю.Д., Купрюшин А.С. Влияние местоположения и использования удерживающих устройств на особенности детской автомобильной травмы в салоне. Известия высших учебных заведений. Поволжский регион. Медицинские науки. 2017;43(3):57-65. https://doi. org/10.21685/2072-3032-2017-3-7

7. World Health Organization. Global Status Report on Road Safety 2013. World Health Organization; Geneva, Switzerland: 2015. [Accessed on 12 Dec 2020].

8. Fridman L, Fraser-Thomas JL, Pike I, Macpherson AK. Canadian child safety report card: a comparison of injury prevention practices across provinces. Injury Prevention. 2019; 25:252-257. https://doi.org/10.1136/injuryprev-2018-042745.

9. Brubacher J, Desapriya E, Erdelyi S, Chan H. The impact of child safety restraint legislation on child injuries in police-reported motor vehicle collisions in British Columbia: an interrupted time series analysis. Paediatr Child Health. 2016;21(4): e27-31.

10. Arkader, A., Warner, W. C., Tolo, V. T., Sponseller, P. D., \& Skaggs, D. L. (2011). Pediatric Chance Fractures. Journal of Pediatric Orthopaedics, 31(7), 741-744.

11. Institute for Health Metrics and Evaluation (IHME). GBD Compare Data Visualization. Seattle: IHME, University of Washington, 2016. Available from http:// vizhub.healthdata.org/gbd-compare. [Accessed 06 December 2018].

12. Mukherjee, S., Beck, C., Yoganandan, N., \& Rao, R. D. (2016). Incidence and mechanism of neurological deficit after thoracolumbar fractures sustained in motor vehicle collisions. Journal of Neurosurgery: Spine, 24(2), 323-331.

13. Müller, C. W., Otte, D., Decker, S., Stübig, T., Panzica, M., Krettek, C., \& Brand, S. (2014). Vertebral fractures in motor vehicle accidents-a medical and technical analysis of 33,015 injured front-seat occupants. Accident Analysis \& Prevention, 66, 15-19.

14. Beckmann N.M, Chinapuvvula N.R, Zhang X, West 0.C. Epidemiology and Imaging Classification of Pediatric Cervical Spine Injuries: 12-Year Experience at a Level 1 Trauma Center. AJR Am J Roentgenol. 2020;214(6): 1359-1368.

15. Савенкова Е.Н., Ефимов А.А. Детский дорожно-транспортный травматизм как судебно-медицинская проблема. Судебно-медицинская экспертиза. 2017;60(6): 13-17. https://doi.org/10.17116/sudmed201760613-17

16. Бережнова ТА, Кулинцова ЯВ. Современная диагностика неотложных состояний у детей, пострадавших в ДТП. 3доровье и образование. 2016;2: 426428

17. Баиндурашвили АГ, Виссарионов СВ, Павлов ИВ, Кокушин ДН., Леин ГА. Консервативное лечение детей с компрессионными переломами позвонков грудной и поясничной локализации в Российской Федерации (обзор литературы). Ортопедия, Травматология и Восстановительная Хирургия Детского Возраста. 2016;4(1): 48-56. doi:10.17816/PTORS4148-

18. Pigolkin Yul, Sedych EP, Mosoyan AS. Forensic medical assessment of vertebral fractures as a result of a car accident. III Japanese-Russian International Conference on Socially Significant Human Diseases: Medical, Environmental and Technical Problems, and these Silition. Obihiro, Japan; 2016. p.124.

19. Седых ЕП. Судебно-медицинская оценка повреждений позвоночника мозга при травме в салоне движущегося легкового автомобиля, оборудованного современными средствами индивидуальной безопасности. Медицинская экспертиза и право. 2015;

20. Liu G, Chen S, Zeng Z, Cui H, Fang Y, Gu D. Risk factors for extremely serious road accidents: Results from national Road Accident Statistical Annual Report of China. PLOSONE. 2018;13(8): e0201587. https://doi.org/10.1371/journal.pone.0201587

21. National Highway Traffic Safety Administration. Traffic safety facts, 2017 data: occupant protection. Washington, DC: US Department of Transportation, National Highway Traffic Safety Administration; 2019. Available at https://crashstats.nhtsa.dot.gov/Api/Public/ViewPublication/812719 external icon [Accessed 03 Dec 2020]

22. Poorman GW, Segreto FA, Beaubrun BM, Jalai CM, Horn SR, Bortz CA, Diebo BG, Vira S, Bono 0J, DE LA Garza-Ramos R, Moon JY, Wang C, Hirsch BP, Tishelman JC, Zhou PL, Gerling M, Passias PG. Traumatic Fracture of the Pediatric Cervical Spine: Etiology, Epidemiology, Concurrent Injuries, and an Analysis of Perioperative Outcomes Using the Kids Inpatient Database. Int J Spine Surg. 2019;13(1): 68-78.

23. Beckmann NM, Chinapuvvula NR, Zhang X, West OC. Epidemiology and Imaging Classification of Pediatric Cervical Spine Injuries: 12-Year Experience at a Level 1 Trauma Center. AJR Am J Roentgenol. 2020;214(6): 1359-1368.

24. Motor vehicle crash-related injury causation scenarios for spinal injuries in restrained children and adolescents. Zonfrillo MR, Locey CM, Scarfone SR, Arbogast KB. 2015

25. Miriam Santschi, MD; Vincent Echavé, MD; Sophie Laflamme, MD; Nathalie McFadden, MD; Claude Cyr, MD. Seat-belt injuries in children involved in motor vehicle crashes. Candian Jurnal of Surger, Vol. 48, No. 5, 0ctober 2005 373-376.

26. Рассоха В.И., Исхаков М.М. Повышение эффективности детских автомобильных кресел (удерживающих устройств). Вестник НЦ БЖД. $2011 ; 4: 27-31$. 
27. Grivna, M., Eid, H. 0., \& Abu-Zidan, F. M. (2015). Epidemiology of spinal injuries in the United Arab Emirates. World Journal of Emergency Surgery: WJES, 10, 20. https://doi.org/10.1186/s13017-015-0015-8

28. Lenehan, B, Boran, S., Street, J., Higgins, T., McCormack, D., \& Poynton, A. R. (2009). Demographics of acute admissions to a National Spinal Injuries Unit. European Spine Journal: Official Publication of the European Spine Society, the European Spinal Deformity Society, and the European Section of the Cervical Spine Research Society, 18(7), 938-942. https://doi.org/10.1007/s00586-009-0923-y

29. Roche, S. J., Sloane, P. A., \& McCabe, J. P. (2008). Epidemiology of spine trauma in an Irish regional trauma unit: a 4-year study. Injury, 39(4), 436-442. https:// doi.org/ 10.1016/j.injury.2007.12.012

30. Tee, J. W., Chan, C. H. P., Fitzgerald, M. C. B., Liew, S. M., \& Rosenfeld, J. V. (2013). Epidemiological trends of spine trauma: an Australian level 1 trauma centre study. Global Spine Journal, 3(2), 75-84. https://doi.org/10.1055/s-0033-1337124

31. Сведения о показателях состояния безопасности дорожного движения (архив) [Электронный ресурc]. URL: http://www.gibdd.ru/stat/archive/ (дата0бращения: 22.03.2018)

32. Официальный сайт Госавтоинспекции МВД России. Ссылка активна на 21.02.18. Доступно по: http://stat.gibdd.ru/ Availableat: http://stat.gibdd.ru/

33. Lindsay M. Andras, MD, Kira F. Skaggs, BA, Haleh Badkoobehi, MD, Paul D. Choi, MD, and David L. Skaggs, MD, MMM (2019) Chance Fractures in the Pediatric Population are Often Misdiagnosed. Journal of Pediatric Orthopaedics: May/June 2019 — Volume 39 — Issue 5 — p 222-225.

34. Miriam Santschi, MD; Vincent Echavé, MD; Sophie Laflamme, MD; Nathalie McFadden, MD; Claude Cyr, MD (2005). Seat-belt injuries in children involved in motor vehicle crashes. Ссылка: Seat-belt injuries in children involved in motor vehicle crashes — PubMed (nih.gov). Ссылка активна на 16.06.2021.

35. Andrew J. Grossbach, M.D., Nader S. Dahdaleh, M.D., Taylor J. Abel, M.D., Gregory D. Woods, B.S., Brian J. Dlouhy, M.D.,1 and Patrick W. Hitchon, M.D. (2013). Flexion-distraction injuries of the thoracolumbar spine: open fusion versus percutaneous pedicle screw fixation. Neurosurg Focus - Volume 35 - August $2013-p 1-6$.

36. Poorman GW, Segreto FA, Beaubrun BM, Jalai CM, Horn SR, Bortz CA, Diebo BG, Vira S, Bono 0J, DE LA Garza-Ramos R, Moon JY, Wang C, Hirsch BP, Tishelman JC, Zhou PL, Gerling M, Passias PG. Traumatic Fracture of the Pediatric Cervical Spine: Etiology, Epidemiology, Concurrent Injuries, and an Analysis of Perioperative Outcomes Using the Kids' Inpatient Database. Int J Spine Surg. 2019 Jan;13(1):68-78.

37. DeVivo M.J. Epidemiology of traumatic spinal cord injury: trends and future implications // Spinal Cord. — 2012. — Vol. 50. — P. 365-372.

38. Zonfrillo MR, Durbin DR, Winston FK, Zhao H, Stineman MG. Physical disability after injury-related inpatient rehabilitation in children. Pediatrics. 2013; 131: e206-213. [PubMed: 23248228].

39. Adel Elkbuli, MD, MPH, Brianna Dowd, Paul J. Spano II, MS, Mark Mc Kenney, MD, MBA. Pediatric Seat Belt Use in Motor Vehicle Collisions: The Need for Driver Education Programs. JOURNAL OF TRAUMA NURSING 2020; Volume 27, Number 5.

40. А.Г. Лебедев, Т.П. Македонская, А.В. Сачков, Н.В. Шаврина, О.Х. Калоева, В.Д. Левитский, П.А. Ярцев. Гнойно-воспалительные осложнения повреждений брюшной стенки от автомобильного ремня безопасности. Опыт лечения четырех пациентов. Russian Sklifosovsky Journal of Emergency Medical Care. 2019; 8(2): 194-202. D0I: 10.23934/2223-9022-2019-8-2-194-202

41. Ахадов Т.А., Мельников И.А., Исхаков 0.С., Божко 0.В., Костикова Т.Д., Манжурцев А.В., Ублинский М.В., Мещеряков С.В., Максумов А.А. Мультиспиральная компьютерная и магнитно-резонансная томография тораколюмбальной травмы позвоночника у детей. Детская хирургия. 2020; 24(5): 323-330. (In Russian). D0l: https://dx.doi.org/10.18821/1560-9510-2020-24-5-323-330

(c) Максумов Александр Алишерович (dr.maksumov@gmail.com ),

Исхаков Олимджан Садыкович, Мещеряков Семен Владимирович.

Журнал «Современная наука: актуальные проблемы теории и практики» 